\title{
Synchronous Analysis for Fuzzy Coupled Neural Networks with Column Pinning Controllers
}

\author{
Dawei Gong $\mathbb{D}^{1},{ }^{1}$ Shijie Song, ${ }^{1}$ Michel Lopez, ${ }^{2}$ and Edgar N. Sanchez ${ }^{2}$ \\ ${ }^{1}$ Mechatronics Engineering of University of Electronic Science and Technology of China, Chengdu 611731, China \\ ${ }^{2}$ Advanced Studies and Research Center of the National Polytechnic Institute, Guadalajara 07360, Mexico \\ Correspondence should be addressed to Dawei Gong; pzhzhx@126.com
}

Received 9 July 2020; Accepted 16 September 2020; Published 5 October 2020

Academic Editor: Yanan Li

Copyright (c) 2020 Dawei Gong et al. This is an open access article distributed under the Creative Commons Attribution License, which permits unrestricted use, distribution, and reproduction in any medium, provided the original work is properly cited.

The synchronous research for fuzzy coupled neural networks (FCNNs) is studied by a new strategy of column pinning controllers. In this paper, the Lyapunov Krasovskii functional (LKF) is taken as an important element for the pinning control laws. The networks are interconnected by coupling gains that define a physical interaction graph. Different from the preset technique in traditional intermittent control, a novel additional communication control graphs of pinning control law are introduced, which has not been investigated before. The proposed control laws can achieve the control objectives of being introduced as an array of vector with Kronecker produce operation. Under the proposed framework of intermittent control, numerical simulations via MATLAB are used to confirm the availability of the suggested control laws.

\section{Introduction}

In recent decades, the investigation on neural networks (NNS) has aroused ever-increasing interest of researchers due to their strong application in various fields [1-5]. The coupled neural networks (CNNs) are seen as a special type of complex networks, which consist of a large set of interconnected single NNs with each individual being called node. Usually, the CNNs exhibit more unpredictable and complicated behaviors than the single NNs. Synchronization of CNNs describes a typical collective behavior and has many applications. For example, the complex oscillatory patterns were stored and retrieved as the synchronization states by presenting an architecture of CNNs in [6,7]. A secure communication system was introduced by utilizing the coupled cellular NNs in [8]. The research on synchronization of CNNs not only opens up new opportunities in the understanding of brain science but also makes an important step forward to the practical applications [9].

However, the aforementioned results are valid when for the structures and the parameters of coupled neural networks are exactly known. In many practical models of the real world, uncertainty or vagueness is unavoidable. Fuzzy theory [10-12] is considered an efficient tool to solve vagueness problems of the complex systems. Compared with the traditional NNS, the FCNNS have advantages for their capabilities in handling uncertain information and representing nonlinear dynamics [13-15].

In practice, many feasible control schemes can be adopted to study synchronous research of complex networks, such as the sampled-data control [16] and intermittent control [17]. Among them, the sampled-data control and impulsive control are two schemes with low control cost because their controllers are updated only at some discrete times. Besides, intermittent control is also an economic choice. In such scheme, the controller is only imposed on the systems at work time. Hence, the notion of intermittent control came into researchers' vision and has stimulated many renewed results. In [18], the quasi-synchronization of delayed chaotic systems was investigated by periodically intermittent control. In [19], the synchronization issues of complex networks were visited by designing an intermittent controller equipped with two switched periods. There are two categories of synchronization: self-synchronization and forced synchronization. Without any external force, the selfsynchronization can be achieved by the connection of local 
nodes. However, the networks usually cannot be synchronized by themselves. Therefore, it is more desirable to force the networks to synchronize. Due to the high dimension and complex topology, it will be expensive and literally infeasible to add controllers to all nodes. Hinted by such consideration, the strategy so-called pinning control is proposed which only controls a small location of the nodes, such as [20-24].

As far as we know, there are no pining control results for FCNNs. So, how to solve the pinning synchronization problems for FCNNs is still challenging. Motivated by the foregoing discussion, this brief explores the synchronization of FCNNs by proposing the concept of column pinning control law. In the developed control scheme, the work conditions are decided by the dynamic relationships among the Lyapunov-Krasovskii functional (LKF) and some other column vectors. Namely, the pinning controller is imposed on the systems when the trajectory of LKF goes into the column regions. Our scheme changes the intrinsic characteristic of the existing control methods that the work conditions are predetermined in prior. From the events' point of view, whether the controller is imposed or not is decided by the dynamic of LKF. Therefore, our scheme can be understood as a class of event-dependent column controllers. Under the framework of the proposed scheme, several simple criterions are developed to study the synchronization for the considered FCNNs.

Notations: $N, R^{n \times m}$, and $R^{n}$ denote the sets of nonnegative integers, $n \times m$ real matrices, and $n$-dimensional Euclidean space, respectively. For real symmetric matrix $\Upsilon$, $\Upsilon>0(\Upsilon \geq 0)$ indicates that $\Upsilon$ is positive definite (respectively, semidefinite). The superscript $T$ stands for the transpose of a matrix. $I_{n}$ denotes the $n$-dimensional identity matrix. $\operatorname{diag}(\cdots)$ represents the block-diagonal matrix.

\section{Problem Formulation}

Without the loss of generality, this brief considers the following FCNNs with $N$ identical nodes:

$$
\begin{aligned}
& \left.\dot{z}_{i}(t)=-C z_{i}(t)+A f\left(z_{i}(t)\right)+B f\left(z_{i}(t-\tau(t))\right)+\sum_{j=1}^{N} G_{i j}^{(1)} D_{1} z_{j}(t)\right)+\sum_{j=1}^{N} G_{i j}^{(2)} D_{2} z_{j}(t-\tau(t))+u_{i}(t), \quad i=1,2, \ldots, h, \\
& \left.\dot{z}_{i}(t)=-C z_{i}(t)+A f\left(z_{i}(t)\right)+B f\left(z_{i}(t-\tau(t))\right)+\sum_{j=1}^{N} G_{i j}^{(1)} D_{1} z_{j}(t)\right)+\sum_{j=1}^{N} G_{i j}^{(2)} D_{2} z_{j}(t-\tau(t)), \quad i=h+1, h+2, \ldots, N,
\end{aligned}
$$

in the formula, $z_{i}(t)=\left(z_{i 1}(t), z_{i 2}(t), \ldots, z_{\text {in }}(t)\right)^{T} \in R^{n}$, $A \in R^{n \times n}, B \in R^{n \times n}, f\left(z_{i}(t)\right)=\left(f_{1}\left(z_{i 1}(t)\right), f_{2}\left(z_{i 2}(t)\right), \ldots\right.$, $\left.f_{n}\left(z_{\text {in }}(t)\right)\right)^{T}, C=\operatorname{diag}\left(c_{1}, c_{2}, \ldots, c_{n}\right)$ are diagonal positive matrix, $G^{(1)}=\left(G_{i j}^{(1)}\right)_{N \times N}, G^{(2)}=\left(G_{i j}^{(2)}\right)_{N \times N}$ are the outer coupled matrix, and $D_{1} \in R^{n \times n}, D_{2} \in R^{n \times n}$ are the inner coupled matrix.

$\tau(t)$ satisfied

$$
0 \leq \dot{\tau}(t) \leq \mu<1,0 \leq \tau(t) \leq \tau,
$$

where $\mu$ and $\tau$ are known constants.

$u_{i}(t)$ are the pinning controllers.

The controllers are designed as

$$
\begin{aligned}
u_{i}(t)= & \sum_{j=1, j \neq i}^{N} k_{i j}^{(1)} D_{3}\left(z_{j}(t)-z_{i}(t)\right) \\
& +\sum_{j=1, j \neq i}^{N} k_{i j}^{(2)} D_{4}\left(z_{j}(t-\tau(t))-z_{i}(t-\tau(t))\right),
\end{aligned}
$$

where $k_{i j}^{(q)}>0,(q=1,2), i=1,2, \ldots, h$, and $k_{i j}^{(q)}=0$, for $i=h+1, h+2, \ldots, N$, and $k_{i j}^{(q)}$ are the control graph weights. Matrices $D_{3}, D_{4} \in R^{n \times n}$ represent control gain matrices. These gain matrices are the control parameters designed to guarantee synchronization of the coupled neural networks.

Remark 1. It is noted that the physical coupling graphs combined with the communication control graphs together form a cyber-physical system, where in the physical connection graph topology $G_{i j}^{(1)}$ and $G_{i j}^{(2)}$ and the communication connection graph topology $k_{i j}^{(1)}$ and $k_{i j}^{(2)}$ are fixed. The design freedom is in the selection of the control gain matrices $D_{3}$ and $D_{4}$.

System (3) can be rewritten as

$$
u_{i}(t)=\sum_{j=1}^{N} L_{i j}^{(1)} D_{3} z_{j}(t)+\sum_{j=1}^{N} L_{i j}^{(2)} D_{4} z_{j}(t-\tau(t)),
$$

where matrix $L^{(q)}=\left(L_{i j}^{(q)}\right)_{N \times N},(q=1,2)$, satisfied

$$
\begin{cases}L_{i j}^{(q)}=k_{i j}^{(q)}, & i \neq j, \\ L_{i i}^{(q)}=-\sum_{j=1, j \neq i}^{N} k_{i j}^{(q)}, & i, j=1,2, \ldots, N .\end{cases}
$$

The initial variables are given as

$$
\begin{aligned}
& z_{i}(s)=\Pi_{i 0}(s) \in \Theta\left([-\tau, 0], R^{n}\right), \quad i=1,2, \ldots, N . \\
& \text { Let } \\
& z(t)=\left(z_{1}^{T}(t), z_{2}^{T}(t), \ldots, z_{N}^{T}(t)\right)^{T}, \\
& F(z(t))=\left(f^{T}\left(z_{1}(t)\right), f^{T}\left(z_{2}(t)\right), \ldots, f^{T}\left(z_{N}(t)\right)\right)^{T} \text {, } \\
& \widetilde{U}(t)=\left(u_{1}^{T}(t), u_{2}^{T}(t), \ldots, u_{N}^{T}(t)\right)^{T} .
\end{aligned}
$$

Combining with the sign $\otimes$ of Kronecker product, system (1) can be rewritten as 


$$
\begin{aligned}
\dot{z}(t)= & -\left(I_{N} \otimes C\right) z(t)+\left(I_{N} \otimes A\right) F(z(t)) \\
& +\left(I_{N} \otimes B\right) F(z(t-\tau(t)))+\left(G^{(1)} \otimes D_{1}\right) z(t) \\
& +\left(G^{(2)} \otimes D_{2}\right) z(t-\tau(t))+\widetilde{U}(t) .
\end{aligned}
$$

From equation (15), we have

$$
\widetilde{U}(t)=\left(L^{(1)} \otimes D_{3}\right) z(t)+\left(L^{(2)} \otimes D_{4}\right) z(t-\tau(t)) .
$$

Remark 2. It is the first introduction of the pinning control laws as an array of vector with Kronecker produce operation.

Assumption 1 (see [25-27]). The outer-coupling matrix are assumed as

$$
\begin{cases}G_{i j}^{(q)}=G_{j i}^{(q)} \geq 0, & i \neq j, q=1,2, \\ G_{i i}^{(q)}=-\sum_{j=1, j \neq i}^{N} G_{i j}^{(q)}, & i, j=1,2, \ldots, N .\end{cases}
$$

Assumption 2 (see [28-30]). For $j \in 1,2, \ldots, N, \forall s_{1}, s_{2} \in R$, $s_{1} \neq s_{2}$, the neural activation functions satisfy

$$
\sigma_{r}^{-} \leq \frac{f_{j}\left(s_{1}\right)-f_{j}\left(s_{2}\right)}{s_{1}-s_{2}} \leq \sigma_{r}^{+} .
$$

We define

$$
\begin{aligned}
& \Delta_{1}=\operatorname{diag}\left(\sigma_{1}^{+} \sigma_{1}^{-}, \ldots, \sigma_{n}^{+} \sigma_{n}^{-}\right), \\
& \Delta_{2}=\operatorname{diag}\left(\frac{\sigma_{1}^{+}+\sigma_{1}^{-}}{2}, \ldots, \frac{\sigma_{n}^{+}+\sigma_{n}^{-}}{2}\right) .
\end{aligned}
$$

From T-S fuzzy model concept, for the first time, a class of FNNS with pinning controllers is described here. Model 1 with T-S theory is described.

Rule $l$ : if $\theta_{1}(t)$ is $F_{l 1}, \theta_{2}(t)$ is $F_{l 2}, \ldots, \theta_{g}(t)$ is $F_{l g}$, then

$$
\begin{aligned}
& \left.\dot{z}_{i}(t)=-C_{l} z_{i}(t)+A_{l} f\left(z_{i}(t)\right)+B_{l} f\left(z_{i}(t-\tau(t))\right)+\sum_{j=1}^{N} G_{i j}^{(1)} D_{1 l} z_{j}(t)\right)+\sum_{j=1}^{N} G_{i j}^{(2)} D_{2 l} z_{j}(t-\tau(t))+u_{i}(t), \quad i=1,2, \ldots, h, \\
& \left.\dot{z}_{i}(t)=-C_{l} z_{i}(t)+A_{l} f\left(z_{i}(t)\right)+B_{l} f\left(z_{i}(t-\tau(t))\right)+\sum_{j=1}^{N} G_{i j}^{(1)} D_{1 l} z_{j}(t)\right)+\sum_{j=1}^{N} G_{i j}^{(2)} D_{2 l} z_{j}(t-\tau(t)), \quad i=h+1, h+2, \ldots, N .
\end{aligned}
$$
form

The controllers of the fuzzy systems are assumed in the

$$
\begin{aligned}
u_{i}(t)= & \sum_{j=1, j \neq i}^{N} k_{i j}^{(1)} D_{3 l}\left(z_{j}(t)-z_{i}(t)\right) \\
& +\sum_{j=1, j \neq i}^{N} k_{i j}^{(2)} D_{4 l}\left(z_{j}(t-\tau(t))-z_{i}(t-\tau(t))\right) .
\end{aligned}
$$

Controller (14) can be rewritten as

$$
u_{i}(t)=\sum_{j=1}^{N} L_{i j}^{(1)} D_{3 l} z_{j}(t)+\sum_{j=1}^{N} L_{i j}^{(2)} D_{4 l} z_{j}(t-\tau(t))
$$

where matrix $L^{(q)}=\left(L_{i j}^{(q)}\right)_{N \times N},(q=1,2)$ are defined as

$$
\begin{cases}L_{i j}^{(q)}=k_{i j}^{(q)}, & i \neq j, \\ L_{i i}^{(q)}=-\sum_{j=1, j \neq i}^{N} k_{i j}^{(q)}, & i, j=1,2, \ldots, N .\end{cases}
$$

The sign of $\otimes$ is used to replace the Kronecker product, and FCNNs system 13 can be expressed as

$$
\begin{aligned}
\dot{z}(t)= & \sum_{l=1}^{r} \mu_{l}(\theta(t))\left(-\left(I_{N} \otimes C_{l}\right) z(t)+\left(I_{N} \otimes A_{l}\right) F(z(t))\right. \\
& +\left(I_{N} \otimes B_{l}\right) F(z(t-\tau(t)))+\left(G^{(1)} \otimes D_{1 l}\right) z(t) \\
& \left.+\left(G^{(2)} \otimes D_{2 l}\right) z(t-\tau(t))\right)+\tilde{U}(t),
\end{aligned}
$$

$\theta(t)=\left[\theta_{1}(t), \theta_{2}(t), \ldots, \theta_{g}(t)\right], \quad \mu_{l}(\theta(t))=\left(\omega_{l}(\theta(t)) / \sum_{l=1}^{r}\right.$ $\left.\omega_{l}(\theta(t))\right), F_{l j}\left(\theta_{j}(t)\right)$ is the grade of membership of $\theta_{j}(t)$ in $F_{l j} \cdot \mu_{l}(\theta(t))$ satisfied

$$
\begin{aligned}
\mu_{l}(\theta(t)) & \geq 0, \\
\sum_{l=1}^{r} \mu_{l}(\theta(t)) & =1 .
\end{aligned}
$$

The controllers of a set of fuzzy rules are written as follows.

Rule $l$ : If $\theta_{1}(t)$ is $F_{l 1}, \theta_{2}(t)$ is $F_{l 2}, \ldots, \theta_{g}(t)$ is $F_{l g}$, then

$$
\begin{array}{r}
\tilde{U}(t)=\left(L^{(1)} \otimes D_{3 l}\right) z(t)+\left(L^{(2)} \otimes D_{4 l}\right) z(t-\tau(t)), \\
l=1,2, \ldots, r .
\end{array}
$$

The resulting FCNNs system can be rewritten as 


$$
\begin{aligned}
\dot{z}(t)= & \sum_{l=1}^{r} \sum_{k=1}^{r} \mu_{l}(\theta(t)) \mu_{k}(\theta(t))\left(-\left(I_{N} \otimes C_{l}\right) z(t)\right. \\
& +\left(I_{N} \otimes A_{l}\right) F(z(t))+\left(I_{N} \otimes B_{l}\right) F(z(t-\tau(t))) \\
& +\left(G^{(1)} \otimes D_{1 l}\right) z(t)+\left(G^{(2)} \otimes D_{2 l}\right) z(t-\tau(t)) \\
& \left.+\left(L^{(1)} \otimes D_{3 k}\right) z(t)+\left(L^{(2)} \otimes D_{4 k}\right) z(t-\tau(t))\right) .
\end{aligned}
$$

Then, we will introduce some useful situations, which are very important to prove our main results.

Definition 1. System (17) is synchronized if the following equation holds:

$$
\lim _{t \longrightarrow \infty}\left\|z_{i}(t)-z_{j}(t)\right\|=0, \quad i, j=1,2, \ldots, N .
$$

Lemma 1 (see [31]). Define $E_{N}=e e^{T}$, where $e=(1,1, \ldots$, $1)^{T}$, and $U=N I_{N}-E_{N}, M \in R^{n \times n}, z=\left(z_{1}^{T}, z_{2}^{T}, \ldots, z_{N}^{T}\right)^{T}$, and $x=\left(x_{1}^{T}, x_{2}^{T}, \ldots, x_{N}^{T}\right)^{T}$ with $z_{k}, x_{k} \in R^{n},(k=1,2, \ldots$, $N)$, then

$$
z^{T}(U \otimes M) x=\sum_{1 \leq i<j \leq N}^{N}\left(\left(z_{i}-x_{j}\right)^{T} M\left(z_{i}-x_{j}\right) .\right.
$$

Lemma 2 (Jensen's inequality). For any real matric $\Theta \in R^{n \times n}, \Theta^{T}=\Theta>0$, constant $\varrho>0$ and $\omega:[0, \varrho] \longrightarrow R^{n}$, then

$$
\varrho \int_{0}^{\varrho} \omega^{T}(s) \Theta \omega(s) \mathrm{d} s \geq\left(\int_{0}^{\varrho} \omega(s) \mathrm{d} s\right)^{T} \Theta\left(\int_{0}^{\varrho} \omega(s) \mathrm{d} s\right) .
$$

Lemma 3 (see [32]). For symmetric constant matric $\Theta=\left[\begin{array}{ccc}\Theta_{11} & \Theta_{12} & \Theta_{13} \\ * & \Theta_{22} & \Theta_{23} \\ * & * & \Theta_{33}\end{array}\right]>0, \quad \Theta_{k q} \in R^{n \times n}, 1 \leq q \leq 3,1 \leq k \leq 3$, $0<\tau(t) \leq \tau$, and vector function $\dot{z}(t):[-\tau(t), 0] \longrightarrow R^{n \times N}$, then we have

$$
\begin{aligned}
& -\tau(t) \int_{t-\tau(t)}^{t}\left[\begin{array}{c}
z(s) \\
F(z(s)) \\
\dot{z}(s)
\end{array}\right]^{T}\left[\begin{array}{ccc}
U \otimes \Theta_{11} & U \otimes \Theta_{12} & U \otimes \Theta_{13} \\
* & U \otimes \Theta_{22} & U \otimes \Theta_{23} \\
* & * & U \otimes \Theta_{33}
\end{array}\right] \\
& \quad\left[\begin{array}{c}
z(s) \\
F(z(s)) \\
\dot{z}(s)
\end{array}\right] \mathrm{d} s, \\
& \leq-\psi^{T}(t)\left[\begin{array}{cccc}
U \otimes \Theta_{11} & U \otimes \Theta_{12} & U \otimes \Theta_{13} & -U \otimes \Theta_{13} \\
* & U \otimes \Theta_{22} & U \otimes \Theta_{23} & -U \otimes \Theta_{23} \\
* & * & U \otimes \Theta_{33} & -U \otimes \Theta_{33} \\
* & * & * & U \otimes \Theta_{33}
\end{array}\right] \psi(t),
\end{aligned}
$$

where $\quad \psi^{T}(t)=\left[\left(\int_{(t-\tau t)}^{t} z(s) d s\right)^{T},\left(\int_{(t-\tau t)}^{t} F(z(s)) d s\right)^{T}\right.$,
$\left.z^{T}(t), z^{T}(t-\tau(t))\right]$.

\section{Synchronization Results for Fuzzy System}

First, we consider the synchronization results of FCNNs without control. Whereafter, we will establish some concise sufficient conditions which ensure synchronization of FCNNs.

3.1. Synchronization for FNNs without Control. In this section, we first study the synchronization criterions for TFNNS with time-varying delay and hybrid coupling:

$$
\begin{aligned}
\dot{z}(t)= & \sum_{l=1}^{r} \mu_{l}(\theta(t))\left(-\left(I_{N} \otimes C_{l}\right) z(t)+\left(I_{N} \otimes A_{l}\right) F(z(t))\right. \\
& +\left(I_{N} \otimes B_{l}\right) F(z(t-\tau(t)))+\left(G^{(1)} \otimes D_{1 l}\right) z(t) \\
& \left.+\left(G^{(2)} \otimes D_{2 l}\right) z(t-\tau(t))\right) .
\end{aligned}
$$

Theorem 1. For $l=1,2, \ldots, r$, system 25 is synchronized if $P_{z}>0(z=1,2,3)$ and $J_{1}>0, J_{2}>0$, then the following formulas are holding for all $1 \leq i<j \leq N$ :

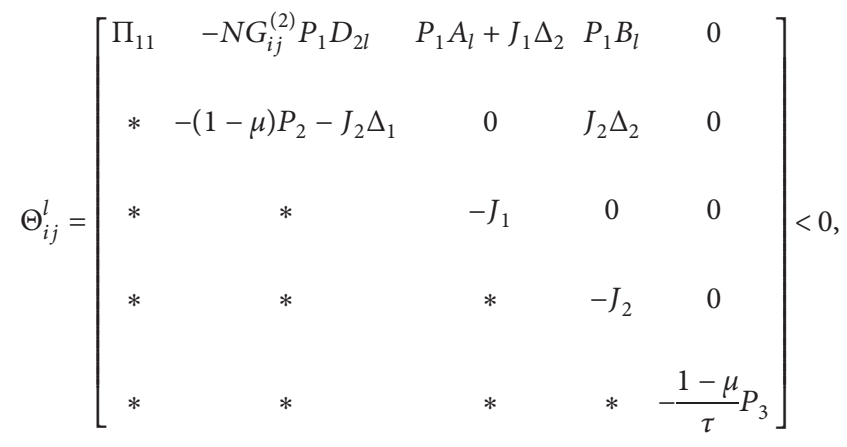

where

$$
\begin{aligned}
\Pi_{11}= & -P_{1} C_{l}-C_{l} P_{1}-J_{1} \Delta_{1}-N G_{i j}^{(1)} P_{1} D_{1 l} \\
& -N G_{i j}^{(1)} D_{1 l}^{T} P_{1}^{T}+P_{2}+\tau P_{3} .
\end{aligned}
$$

Proof. Consider $U$ as Lemma 1; for system (17), we have

$$
\begin{aligned}
& V_{1}(t)=z^{T}(t)\left(U \otimes P_{1}\right) z(t), \\
& V_{2}(t)=\int_{t-\tau(t)}^{t} z^{T}(s)\left(U \otimes P_{2}\right) z(s) \mathrm{d} s, \\
& V_{3}(t)=\int_{t-\tau(t)}^{t} \int_{\theta}^{t} z^{T}(s)\left(U \otimes P_{3}\right) z(s) \mathrm{d} s \mathrm{~d} \theta .
\end{aligned}
$$

Deriving time of system (17), 


$$
\begin{aligned}
\dot{V}_{1}(t)= & 2 z^{T}(t)\left(U \otimes P_{1}\right) \dot{z}(t), \\
= & 2 z^{T}(t)\left(U \otimes P_{1}\right) \sum_{l=1}^{r} \mu_{l}(\theta(t))\left[-\left(I_{N} \otimes C_{l}\right) z(t)+\left(I_{N} \otimes A_{l}\right) F(z(t)),\right. \\
& \left.+\left(I_{N} \otimes B_{l}\right) F(z(t-\tau(t)))+\left(G^{(1)} \otimes D_{1 l}\right) z(t)+\left(G^{(2)} \otimes D_{2 l}\right) z(t-\tau(t))\right], \\
\dot{V}_{2}(t) \leq & z^{T}(t)\left(U \otimes P_{2}\right) z(t)-(1-\mu) z^{T}(t-\tau(t))\left(U \otimes P_{2}\right) z(t-\tau(t)), \\
\dot{V}_{3}(t) \leq & \tau z^{T}(t)\left(U \otimes P_{3}\right) z(t)-(1-\mu) \int_{t-\tau}^{t}\left(z^{T}(s)\right)\left(U \otimes P_{3}\right) z(s) \mathrm{d} s, \\
\leq & \tau z^{T}(t)\left(U \otimes P_{3}\right) z(t)-\frac{1-\mu}{\tau}\left(\int_{t-\tau(t)}^{t} z(s) \mathrm{d} s\right)^{T}\left(U \otimes P_{3}\right)\left(\int_{t-\tau(t)}^{t}(z(s)) \mathrm{d} s\right) .
\end{aligned}
$$

From reference [33] and Assumption 2, for any diagonal matrix $J_{1}, J_{2}$, we have

$$
\begin{aligned}
& 0 \leq\left[\begin{array}{c}
z_{i}(t)-z_{j}(t) \\
f\left(z_{i}(t)\right)-f\left(z_{j}(t)\right)
\end{array}\right]^{T}\left[\begin{array}{rr}
-J_{1} \Delta_{1} & J_{1} \Delta_{2} \\
* & -J_{1}
\end{array}\right]\left[\begin{array}{c}
z_{i}(t)-z_{j}(t) \\
f\left(z_{i}(t)\right)-f\left(z_{j}(t)\right)
\end{array}\right] \\
& +\left[\begin{array}{c}
z_{i}(t-\tau(t))-z_{j}(t-\tau(t)) \\
f\left(z_{i}(t-\tau(t))\right)-f\left(z_{j}(t-\tau(t))\right)
\end{array}\right]\left[\begin{array}{cc}
-J_{2} \Delta_{1} & J_{2} \Delta_{2} \\
* & -J_{2}
\end{array}\right] \\
& \times\left[\begin{array}{c}
z_{i}(t-\tau(t))-z_{j}(t-\tau(t)) \\
f\left(z_{i}(t-\tau(t))\right)-f\left(z_{j}(t-\tau(t))\right)
\end{array}\right]
\end{aligned}
$$

According to (29)-(32), we obtain

$$
\dot{V}(t) \leq \sum_{l=1}^{r} \mu_{l}(\theta(t)) \sum_{i=1}^{N-1} \sum_{j=i+1}^{N}\left(\xi_{i j}^{T}(t) \Theta_{i j}^{l} \xi_{i j}(t)\right) \text {, }
$$

in which $\xi_{i j}(t)=\left(\left(z_{i}(t)-z_{j}(t)\right)^{T},\left(z_{i}(t-\tau(t))-z_{j}(t-\right.\right.$ $\tau(t)))^{T},\left(f\left(z_{i}(t)\right)-f\left(z_{j}(t)\right)\right)^{T},\left(f\left(z_{i}(t-\tau(t))\right)-f\left(z_{j}(t-\right.\right.$ $\tau(t))))^{T},\left(\int_{t-\tau(t)}^{t} z_{i}(s)-z_{j}(s) \mathrm{d} s^{T}\right)^{T}$, and $\Theta_{i j}^{l}$ is the same in Theorem 1. From Definition 1, system (25) is synchronized when $\Theta_{i j}^{l}<0$.
Note that, in Theorem 1, we did not introduce freeweighting matrix. Next, we will choose other Lyanpunov-Krosovskii functional and introduce more freeweighting matrices, which can add more useful conditions.

Theorem 2. For $l=1,2, \ldots, r$, system 25 is synchronized if there exist $P_{q q}>0, Q_{q q}>0, W>0, \quad(q=1,2,3), P_{s q}, Q_{s q}, T$, $(1 \leq s<q \leq 3)$, and $J_{1}>0, J_{2}>0$; then, the following formulas are holding for all $1 \leq i<j \leq N$ : 


$$
\begin{aligned}
& P=\left[\begin{array}{ccc}
P_{11} & P_{12} & P_{13} \\
* & P_{22} & P_{23} \\
* & * & P_{33}
\end{array}\right]>0, \\
& Q=\left[\begin{array}{ccc}
Q_{11} & Q_{12} & Q_{13} \\
* & Q_{22} & Q_{23} \\
* & * & Q_{33}
\end{array}\right]>0,
\end{aligned}
$$



where

$$
\begin{aligned}
& \Xi_{11}=-W C_{l}-C_{l}^{T} W^{T}+P_{11}+\tau Q_{11}-\frac{1-\mu}{\tau} Q_{33}-J_{1} \Delta_{1}, \\
& \Xi_{12}=P_{12}+\tau Q_{12}+W A_{l}-N G_{i j}^{(1)} W D_{1 l}+J_{1} \Delta_{2}, \\
& \Xi_{14}=W B_{l}-N G_{i j}^{(2)} W D_{2 l}, \\
& \Xi_{17}=P_{13}+\tau Q_{13}-C_{l} T^{T}-N G_{i j}^{(1)} D_{1 l}^{T} T^{T}, \\
& \Xi_{22}=P_{22}+\tau Q_{22}-J_{1}, \\
& \Xi_{27}=P_{23}+\tau Q_{23}+A_{l}^{T} T^{T}, \\
& \Xi_{33}=-(1-\mu) P_{11}-\frac{1-\mu}{\tau} Q_{33}-J_{2} \Delta_{1}, \\
& \Xi_{34}=-(1-\mu) P_{12}+J_{2} \Delta_{2}, \\
& \Xi_{44}=-(1-\mu) P_{22}-J_{2}, \\
& \Xi_{77}=P_{33}+\tau Q_{33}-T-T^{T} .
\end{aligned}
$$

Proof. From Assumptions 1 and 2, consider the following LKF for model (17):

$$
V(t)=V_{1}(t)+V_{2}(t)+V_{3}(t)
$$

where

$$
\begin{aligned}
V_{1}(t)= & z^{T}(t)(U \otimes W) z(t), \\
V_{2}(t)= & \int_{t-\tau(t)}^{t}\left[\begin{array}{c}
z(s) \\
F(z(s)) \\
\dot{z}(s)
\end{array}\right]\left[\begin{array}{ccc}
U \otimes P_{11} & U \otimes P_{12} & U \otimes P_{13} \\
* & U \otimes P_{22} & U \otimes P_{23} \\
* & * & U \otimes P_{33}
\end{array}\right] \\
& \cdot\left[\begin{array}{c}
z(s) \\
F(z(s)) \\
\dot{z}(s)
\end{array}\right] \mathrm{d} s, \\
V_{3}(t)= & \left.\int_{t-\tau(t)}^{t}\right]_{\theta}^{t}\left[\begin{array}{c}
z(s) \\
F(z(s)) \\
\dot{z}(s)
\end{array}\right]\left[\begin{array}{ccc}
U \otimes Q_{11} & U \otimes Q_{12} & U \otimes Q_{13} \\
* & U \otimes Q_{22} & U \otimes Q_{23} \\
* & * & U \otimes Q_{33}
\end{array}\right] \\
& \cdot\left[\begin{array}{c}
z(s) \\
F(z(s)) \\
\dot{z}(s)
\end{array}\right] \mathrm{d} s \mathrm{~d} \theta .
\end{aligned}
$$


Calculating the time derivative of system 29 , then

$$
\begin{aligned}
& \dot{V}_{1}(t)=2 z^{T}(t)(U \otimes W) \dot{z}(t), \\
& =2 z^{T}(t)(U \otimes W) \sum_{l=1}^{r} \mu_{l}(\theta(t))\left[-\left(I_{N} \otimes C_{l}\right) z(t)+\left(I_{N} \otimes A_{l}\right) F(z(t),\right. \\
& +\left(I_{N} \otimes B_{l}\right) F(z(t-\tau(t)))+\left(G^{(1)} \otimes D_{1 l}\right) F(z(t)), \\
& \left.+\left(G^{(2)} \otimes D_{2 l}\right) F(z(t-\tau(t)))\right] \\
& \dot{V}_{2}(t)=\left[\begin{array}{c}
z(t) \\
F(z(t)) \\
\dot{z}(t)
\end{array}\right]^{T}\left[\begin{array}{ccc}
U \otimes P_{11} & U \otimes P_{12} & U \otimes P_{13} \\
* & U \otimes P_{22} & U \otimes P_{23} \\
* & * & U \otimes P_{33}
\end{array}\right]\left[\begin{array}{c}
z(t) \\
F(z(t)) \\
\dot{z}(t)
\end{array}\right]-(1-\dot{\tau}(t)), \\
& \times\left[\begin{array}{c}
z(t-\tau(t)) \\
F(z(t-\tau(t))) \\
\dot{z}(t-\tau(t))
\end{array}\right]^{T}\left[\begin{array}{ccc}
U \otimes P_{11} & U \otimes P_{12} & U \otimes P_{13} \\
* & U \otimes P_{22} & U \otimes P_{23} \\
* & * & U \otimes P_{33}
\end{array}\right]\left[\begin{array}{c}
z(t-\tau(t)) \\
F(z(t-\tau(t))) \\
\dot{z}(t-\tau(t))
\end{array}\right] .
\end{aligned}
$$

From Lemmas 2 and 3, we can acquire

$$
\begin{aligned}
& \dot{V}_{3}(t)=\tau(t)\left[\begin{array}{c}
z(t) \\
F(z(t)) \\
\dot{z}(t)
\end{array}\right]^{T}\left[\begin{array}{ccc}
U \otimes Q_{11} & U \otimes Q_{12} & U \otimes Q_{13} \\
* & U \otimes Q_{22} & U \otimes Q_{23} \\
* & * & U \otimes Q_{33}
\end{array}\right]\left[\begin{array}{c}
z(t) \\
F(z(t)) \\
\dot{z}(t)
\end{array}\right], \\
& -(1-\dot{\tau}(t)) \int_{t-\tau(t)}^{t}\left[\begin{array}{c}
z(s) \\
F(z(s)) \\
\dot{z}(s)
\end{array}\right]^{T}\left[\begin{array}{ccc}
U \otimes Q_{11} & U \otimes Q_{12} & U \otimes Q_{13} \\
* & U \otimes Q_{22} & U \otimes Q_{23} \\
* & * & U \otimes Q_{33}
\end{array}\right]\left[\begin{array}{c}
z(s) \\
F(z(s)) \\
\dot{z}(s)
\end{array}\right] \mathrm{d} s, \\
& \leq \tau\left[\begin{array}{c}
z(t) \\
F(z(t)) \\
\dot{z}(t)
\end{array}\right]^{T}\left[\begin{array}{ccc}
U \otimes Q_{11} & U \otimes Q_{12} & U \otimes Q_{13} \\
* & U \otimes Q_{22} & U \otimes Q_{23} \\
* & * & U \otimes Q_{33}
\end{array}\right]\left[\begin{array}{c}
z(t) \\
F(z(t)) \\
\dot{z}(t)
\end{array}\right], \\
& -\frac{1-\mu}{\tau} \psi^{T}(t)\left[\begin{array}{cccc}
U \otimes Q_{11} & U \otimes Q_{12} & U \otimes Q_{13} & -U \otimes Q_{13} \\
* & U \otimes Q_{22} & U \otimes Q_{23} & -U \otimes Q_{23} \\
* & * & U \otimes Q_{33} & -U \otimes Q_{33} \\
* & * & * & U \otimes Q_{33}
\end{array}\right] \psi(t),
\end{aligned}
$$


where $\psi^{T}(t)=\left[\left(\int_{(t-\tau t)}^{t} z(s) \mathrm{d} s\right)^{T}, \quad\left(\int_{(t-\tau t)}^{t} F(z(s)) \mathrm{d} s\right)^{T}, \quad U X=N X\right.$; from Lemma 1, we have $\left.z^{T}(t), z^{T}(t-\tau(t))\right]$.

$$
\begin{aligned}
& \dot{V}_{1}(t)=2 \sum_{i=1}^{N-1} \sum_{j=i+1}^{N}\left(z_{i}(t)-z_{j}(t)\right)^{T} \sum_{l=1}^{r} \mu_{l}(\theta(t))\left[\left(-W C_{l}\right)\left(z_{i}(t)-z_{j}(t)\right)\right. \text {, } \\
& +\left(W A_{l}-N G_{i j}^{(1)} W D_{1 l}\right)\left(f\left(z_{i}(t)\right)-f\left(z_{j}(t)\right)\right), \\
& \left.+\left(W B_{l}-N G_{i j}^{(2)} W D_{2 l}\right)\left(f\left(z_{i}\left(t-\tau_{1}(t)\right)\right)-f\left(z_{j}\left(t-\tau_{1}(t)\right)\right)\right)\right], \\
& \dot{V}_{2}(t) \leq \sum_{i=1}^{N-1} \sum_{j=i+1}^{N}\left[\begin{array}{c}
z_{i}(t)-z_{j}(t) \\
f\left(z_{i}(t)\right)-f\left(z_{j}(t)\right) \\
\dot{z}_{i}(t)-\dot{z}_{j}(t)
\end{array}\right]^{T}\left[\begin{array}{ccc}
P_{11} & P_{12} & P_{13} \\
* & P_{22} & P_{23} \\
* & * & P_{33}
\end{array}\right]\left[\begin{array}{c}
z_{i}(t)-z_{j}(t) \\
f\left(z_{i}(t)\right)-f\left(z_{j}(t)\right) \\
\dot{z}_{i}(t)-\dot{z}_{j}(t)
\end{array}\right], \\
& -(1-\mu) \sum_{i=1}^{N-1} \sum_{j=i+1}^{N}\left[\begin{array}{c}
z_{i}(t-\tau(t))-z_{j}(t-\tau(t)) \\
f\left(z_{i}(t-\tau(t))\right)-f\left(z_{j}(t-\tau(t))\right) \\
\dot{z}_{i}(t-\tau(t))-\dot{z}_{j}(t-\tau(t))
\end{array}\right]^{T}\left[\begin{array}{ccc}
P_{11} & P_{12} & P_{13} \\
* & P_{22} & P_{23} \\
* & * & P_{33}
\end{array}\right] \text {, } \\
& \times\left[\begin{array}{c}
z_{i}(t-\tau(t))-z_{j}(t-\tau(t)) \\
f\left(z_{i}(t-\tau(t))\right)-f\left(z_{j}(t-\tau(t))\right) \\
\dot{z}_{i}(t-\tau(t))-\dot{z}_{j}(t-\tau(t))
\end{array}\right]
\end{aligned}
$$

As the same method, from Lemma 1, we have

$$
\begin{aligned}
& \left.\dot{V}_{3}(t) \leq \tau \sum_{i=1}^{N-1} \sum_{j=i+1}^{N}\left[\begin{array}{c}
z_{i}(t)-z_{j}(t) \\
\dot{z}_{i}(t)-\dot{z}_{j}(t)
\end{array}\right]^{T}(t)\right)-f\left(z_{j}(t)\right)\left[\begin{array}{ccc}
Q_{11} & Q_{12} & Q_{13} \\
* & Q_{22} & Q_{23} \\
* & * & Q_{33}
\end{array}\right]\left[\begin{array}{c}
z_{i}(t)-z_{j}(t) \\
f\left(z_{i}(t)\right)-f\left(z_{j}(t)\right) \\
\dot{z}_{i}(t)-\dot{z}_{j}(t)
\end{array}\right],
\end{aligned}
$$

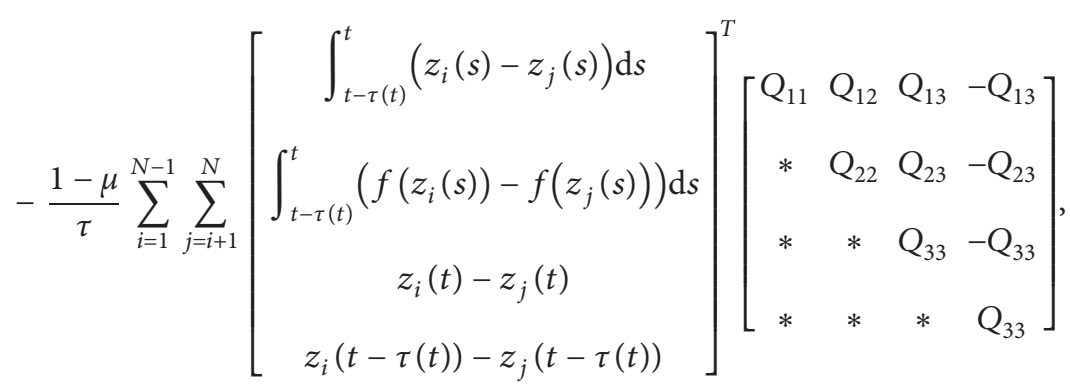

$$
\begin{aligned}
& \times\left[\begin{array}{c}
\int_{t-\tau(t)}^{t}\left(z_{i}(s)-z_{j}(s)\right) \mathrm{d} s \\
\int_{t-\tau(t)}^{t}\left(f\left(z_{i}(s)\right)-f\left(z_{j}(s)\right)\right) \mathrm{d} s \\
z_{i}(t)-z_{j}(t) \\
z_{i}(t-\tau(t))-z_{j}(t-\tau(t))
\end{array}\right] .
\end{aligned}
$$


For any matrix $T \in R^{n \times n}$, from system (17), we can easily obtain

$$
\begin{aligned}
0= & 2 \dot{z}^{T}(t)(U \otimes T) \sum_{l=1}^{r} \mu_{l}(\theta(t))\left(-\dot{z}(t)-\left(I_{N} \otimes C_{l}\right) z(t)\right. \\
& +\left(I_{N} \otimes A_{l}\right) F(z(t)), \\
& +\left(I_{N} \otimes B_{l}\right) F(z(t-\tau(t)))+\left(G^{(1)} \otimes D_{1 l}\right) z(t) \\
& +\left(G^{(2)} \otimes D_{2 l}\right) z(t-\tau(t)) .
\end{aligned}
$$

Let $\quad \zeta_{i j}^{T}(t)=\left(\left(z_{i}(t)-z_{j}(t)\right)^{T}, f\left((z(t))-f\left(z_{j}(t)\right)\right)^{T}\right.$, $\left(z_{i}(t-\tau(t))-z_{j}(t-\tau(t))\right)^{T} f\left(\left(z_{i}(t-\tau(t))\right)-f\left(z_{i}(t-\tau\right.\right.$

$(t))))^{T},\left(\int_{t-\tau(t)}^{t}\left(z_{i}(s)-z_{j}(s)\right) \mathrm{d} s\right)^{T}\left(\int_{t-\tau(t)}^{t}\left(f\left(z_{i}(s)\right)-f\left(z_{j}\right.\right.\right.$ $\left.(s))) \mathrm{d} s)^{T}, \quad\left(\dot{z}_{i}(t)-\dot{z}_{j}(t)\right)^{T},\left(\dot{z}_{i}(t-\tau(t))-\dot{z}_{j}(t-\tau(t))\right)^{T}\right)$, from (32) and (41)-(44), we can obtain

$$
\dot{V}(t) \leq \sum_{l=1}^{r} \mu_{l}(\theta(t)) \sum_{i=1}^{N-1} \sum_{j=i+1}^{N}\left(\zeta_{i j}^{T}(t) \Omega_{i j}^{l} \zeta_{i j}(t)\right),
$$

where $\Omega_{i j}^{l}$ is defined as (50). From Definition 1, it implies that system (17) is synchronized.

3.2. Synchronization for Fuzzy System with Pinning Control. This section deals with the pinning synchronization problems for the closed-loop T-S fuzzy neural networks:

$$
\begin{aligned}
\dot{z}(t)= & \sum_{l=1}^{r} \sum_{k=1}^{r} \mu_{l}(\theta(t)) \mu_{k}(\theta(t))\left(-\left(I_{N} \otimes C_{l}\right) z(t)+\left(I_{N} \otimes A_{l}\right) F(z(t)),\right. \\
& +\left(I_{N} \otimes B_{l}\right) F(z(t-\tau(t)))+\left(G^{(1)} \otimes D_{1 l}\right) z(t)+\left(G^{(2)} \otimes D_{2 l}\right) z(t-\tau(t)), \\
& \left.+\left(L^{(1)} \otimes D_{3 k}\right) z(t)+\left(L^{(2)} \otimes D_{4 k}\right) z(t-\tau(t))\right) .
\end{aligned}
$$

Theorem 3. For $l=1,2, \ldots, r$, dynamical system (46) is synchronized if there is $P_{z}>0(z=2,3,4)$ and positive diagonal matrix $P_{1}, J_{1}$, and $J_{2}$; then, the following formulas are holding for all $1 \leq i<j \leq N$ :

$$
\Theta_{i j}^{l k}=\left[\begin{array}{ccccc}
\Pi_{11} & \Pi_{12} & A_{l} X+X J_{1} X \Delta_{2} & B_{l} X & 0 \\
* & \Pi_{22} & 0 & X J_{2} X \Delta_{2} & 0 \\
* & * & -X J_{1} X & 0 & 0 \\
* & * & * & -X J_{2} X & 0 \\
* & * & * & * & -\frac{1-\mu}{\tau} X P_{3} X
\end{array}\right]<0,
$$

in which

$$
\begin{aligned}
\Pi_{11}= & -C_{l} X-X C_{l}-X J_{1} X \Delta_{1}-N G_{i j}^{(1)} D_{1 l} X-N L_{i j}^{(1)} z_{3 k} \\
& -N G_{i j}^{(1)} X D_{1 l}^{T}-N L_{i j}^{(1)} z_{3 k}^{T}+X P_{2} X+\tau X P_{3} X \\
\Pi_{12}= & -N G_{i j}^{(2)} D_{2 l} X-N L_{i j}^{(2)} z_{4 k}, \\
\Pi_{22}= & -(1-\mu) X P_{2} X-X J_{2} X \Delta_{1} .
\end{aligned}
$$

Proof. Based on Theorem 1, the feedback gains in the fuzzy coupled system are given by $D_{3 k}=z_{3 k} X^{-1}$ and $D_{4 k}=z_{4 k} X^{-1}$. Replace $N G_{i j}^{(1)} D_{3 l}$ with $N G_{i j}^{(1)} D_{1 l}+$ $N L_{i j}^{(1)} D_{3 k}, N G_{i j}^{(2)} D_{2 l}$ with $N G_{i j}^{(2)} D_{4 l}+N L_{i j}^{(2)} D_{4 k}$. Pre- and postmultiply 13 with $\operatorname{diag}[X ; X ; X ; X ; X]$, where $X^{-1}=P_{1}$; then, we can obtain the above criteria.

Theorem 4. For $l=1,2, \ldots, r$, system (46) is synchronized if there exists $P_{q q}>0, Q_{q q}>0, \quad(q=1,2,3), \quad P_{s q}, Q_{s q}$, $(1 \leq s<q \leq 3)$, and $J_{1}>0, J_{2}>0, W>0$; then, the following formulas are holding for all $1 \leq i<j \leq N$ : 


$$
\begin{aligned}
& P=\left[\begin{array}{ccc}
P_{11} & P_{12} & P_{13} \\
* & P_{22} & P_{23} \\
* & * & P_{33}
\end{array}\right]>0, \\
& Q=\left[\begin{array}{ccc}
Q_{11} & Q_{12} & Q_{13} \\
* & Q_{22} & Q_{23} \\
* & * & Q_{33}
\end{array}\right]>0,
\end{aligned}
$$

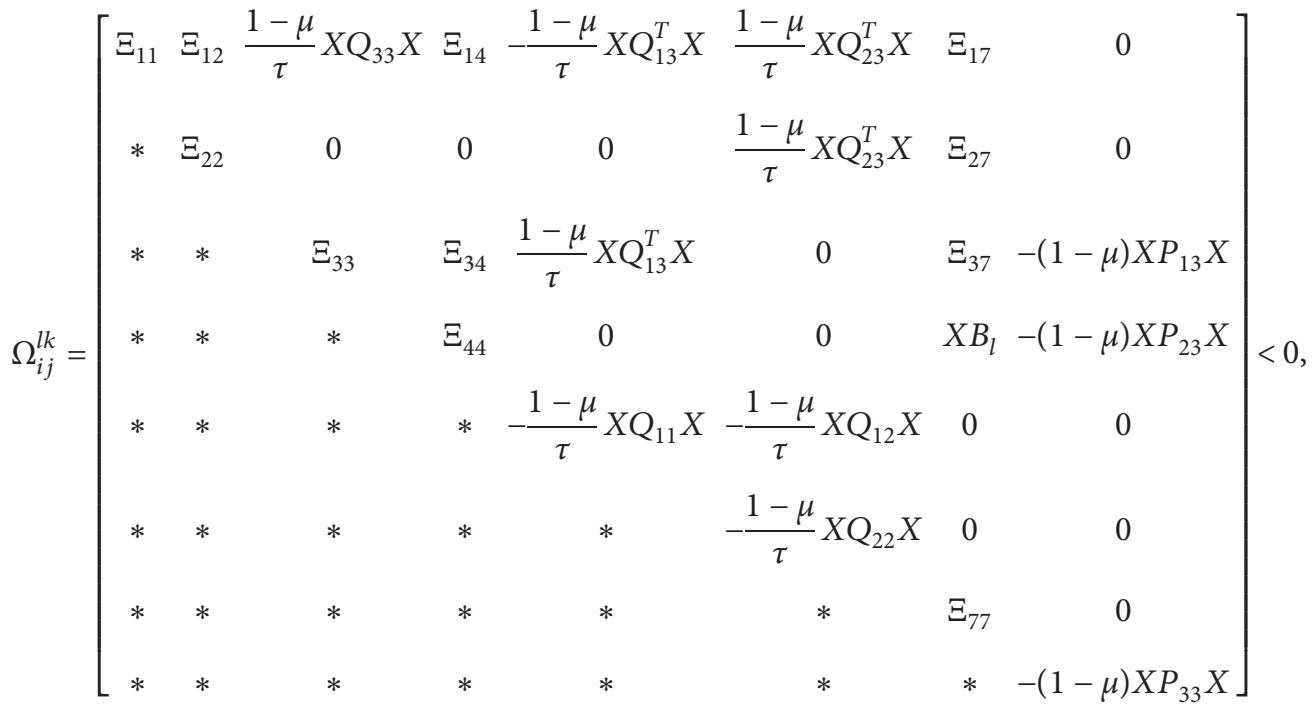

where

$$
\begin{aligned}
\Xi_{11}= & -C_{l} X-X C_{l}^{T}+X P_{11} X+\tau X Q_{11} X \\
& -\frac{1-\mu}{\tau} X Q_{33} X-X J_{1} X \Delta_{1}, \\
\Xi_{12}= & X P_{12} X+\tau X Q_{12} X+A_{l} X-N G_{i j}^{(1)} D_{1 l} X-N L_{i j}^{(1)} z_{3 k} \\
& +X J_{1} X \Delta_{2}, \\
\Xi_{14}= & B_{l} X-N G_{i j}^{(2)} D_{2 l} X-N L_{i j}^{(2)} z_{4 k}, \\
\Xi_{17}= & X P_{13} X+\tau X Q_{13} X-X C_{l}-N G_{i j}^{(1)} X D_{1 l}^{T}-N L_{i j}^{(1)} z_{3 k}^{T}, \\
\Xi_{22}= & X P_{22} X+\tau X Q_{22} X-X J_{1} X, \\
\Xi_{27}= & X P_{23} X+\tau X Q_{23} X+X A_{l}^{T}, \\
\Xi_{33}= & -(1-\mu) X P_{11} X-\frac{1-\mu}{\tau} X Q_{33} X-X J_{2} X \Delta_{1}, \\
\Xi_{34}= & -(1-\mu) X P_{12} X+X J_{2} X \Delta_{2}, \\
\Xi_{37}= & -N G_{i j}^{(1)} X D_{2 l}^{T}-N L_{i j}^{(2)} z_{4 k}^{T}, \\
\Xi_{44}= & -(1-\mu) X P_{22} X-X J_{2} X, \\
\Xi_{77}= & X P_{33} X+\tau X Q_{33} X-X-X .
\end{aligned}
$$

Proof. Based on Theorem 2, let $T=W$, and the feedback gains in the fuzzy system are given by $D_{3 k}=z_{3 k} X^{-1}$ and $D_{4 k}=z_{4 k} X^{-1}$. Replace $N G_{i j}^{(1)} D_{1 l}$ with $N G_{i j}^{(1)} D_{1 l}+N L_{i j}^{(1)} D_{3 k}$, $N G_{i j}^{(2)} D_{2 l}$ with $N G_{i j}^{(2)} D_{2 l}+N L_{i j}^{(2)} D_{4 k}$. Pre- and postmultiply (35) with $\operatorname{diag}[X ; X ; X ; X ; X ; X ; X ; X]$, where $X^{-1}=W$; then, we can obtain the above criteria.

Remark 3. From these two pinning synchronized results, it is noted that the fuzzy pinning control gain matrices $D_{3}$ and $D_{4}$ can be computed, which can fix the communication connection graph topology $k_{i j}^{(1)}$ and $k_{i j}^{(2)}$. Such complex fuzzy pinning controllers are proposed for the first time.

\section{Numerical Examples}

This section provides a numerical example to illustrate the effectiveness of the obtained results. Assume the system without control first and then with pinning control.

4.1. Synchronization for Fuzzy Coupled Networks without Control. Consider the following TNNs model, the parameters in which are defined as 


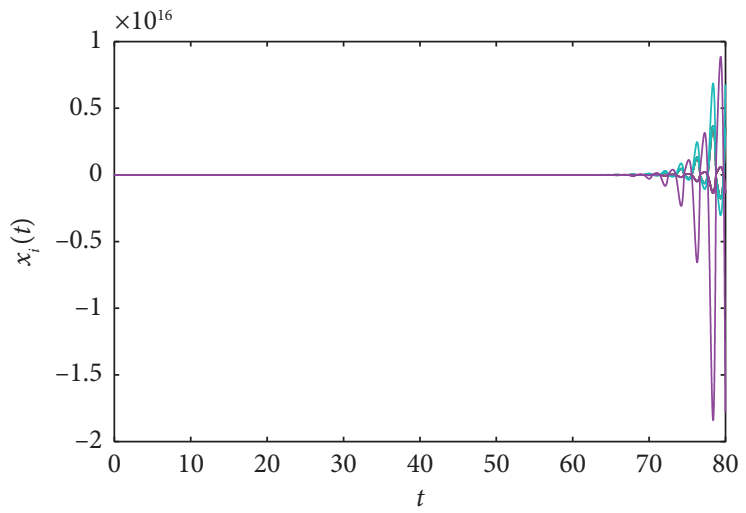

Figure 1: States of fuzzy neural networks (8) without pinning control $\left(L^{(1)}=L^{(2)}=0\right): z_{i}(t), i=1,2,3,4,5,6$.

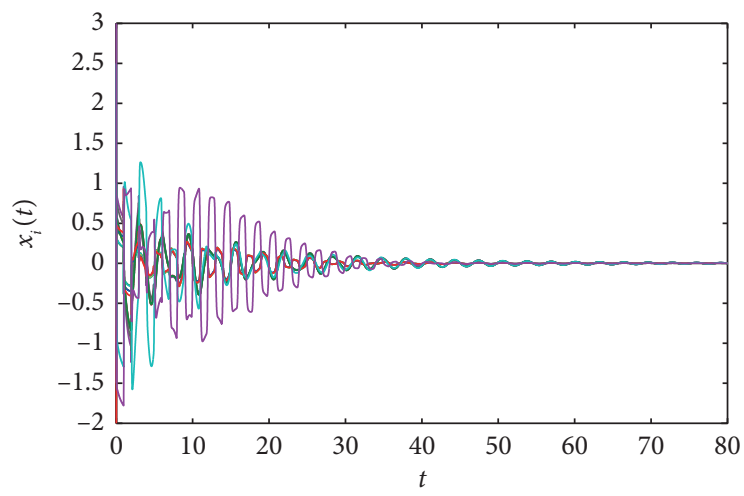

FIGURE 2: States of fuzzy neural networks (8) with pinning control: $z_{i}(t), i=1,2,3,4,5,6$.
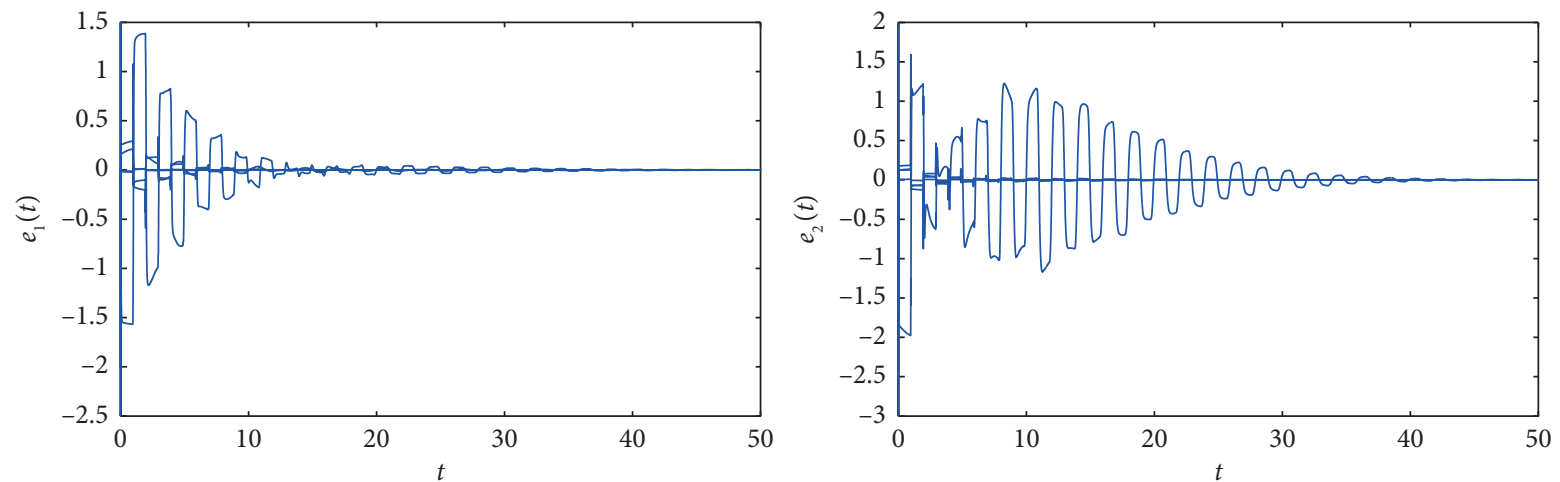

Figure 3: Pinning synchronization errors for fuzzy neural networks: $e_{j}(t), j=1,2$. 


$$
\begin{aligned}
\dot{z}(t)= & \sum_{l=1}^{2} \mu_{l}(\theta(t))\left(-\left(I_{N} \otimes C_{l}\right) z(t)+\left(I_{N} \otimes A_{l}\right) F(z(t))\right. \\
& +\left(I_{N} \otimes B_{l}\right) F(z(t-\tau(t))), \\
& \left.\left.+\left(G^{(1)} \otimes D_{1 l}\right) z(t)+\left(G^{(2)} \otimes D_{2 l}\right) z(t-\tau(t))\right)\right),
\end{aligned}
$$

where

$$
\begin{aligned}
C_{1} & =\left[\begin{array}{ll}
5 & 0 \\
0 & 2
\end{array}\right], C_{2}=\left[\begin{array}{ll}
2 & 0 \\
0 & 1
\end{array}\right], A_{1}=A_{2}=\left[\begin{array}{ll}
1.8 & 10 \\
0.1 & 1.8
\end{array}\right], \\
B_{1} & =B_{2}=\left[\begin{array}{ll}
-1.5 & 0.1 \\
0.1 & -1.5
\end{array}\right], \\
D_{11} & =\left[\begin{array}{ll}
2 & 0 \\
0 & 3
\end{array}\right], D_{12}=\left[\begin{array}{ll}
1 & 0 \\
0 & 1.5
\end{array}\right], D_{21}=\left[\begin{array}{ll}
3 & 1 \\
2 & 1
\end{array}\right], D_{22}=\left[\begin{array}{ll}
3 & 0 \\
0 & 2
\end{array}\right], \\
\mu & =0, \tau=0.97, \mu_{1}=\frac{1}{1+\exp \left(-2 z_{1}(t)\right)}, \mu_{2}=1-\mu_{1} .
\end{aligned}
$$

The outer-coupling matrix $\left(G_{i j}^{(q)}\right)_{N \times N},(q=1,2)$, are defined as

$$
G^{(1)}=G^{(2)}=\left[\begin{array}{cccccc}
-5 & 1 & 0 & 1 & 0 & 3 \\
1 & -5 & 1 & 0 & 0 & 3 \\
0 & 1 & -5 & 0 & 1 & 3 \\
1 & 0 & 0 & -5 & 1 & 3 \\
0 & 0 & 1 & 1 & -5 & 3 \\
3 & 3 & 3 & 3 & 3 & -15
\end{array}\right] .
$$

Then, we plot the states of network (52) without control in Figure 1. It is easy to see that the system cannot be synchronized by itself.

4.2. Pinning Synchronization for Fuzzy Neural Networks with Hybrid Coupling. Now, we consider the system with pinning control:

$$
\begin{aligned}
\dot{z}(t)= & \sum_{l=1}^{r} \sum_{k=1}^{r} \mu_{l}(\theta(t)) \mu_{k}(\theta(t))\left(-\left(I_{N} \otimes C_{l}\right) z(t)\right. \\
& +\left(I_{N} \otimes A_{l}\right) F(z(t)), \\
& +\left(I_{N} \otimes B_{l}\right) F(z(t-\tau(t)))+\left(G^{(1)} \otimes D_{1 l}\right) z(t) \\
& +\left(G^{(2)} \otimes D_{2 l}\right) z(t-\tau(t)), \\
& \left.+\left(L^{(1)} \otimes D_{3 k}\right) z(t)+\left(L^{(2)} \otimes D_{4 k}\right) z(t-\tau(t))\right) .
\end{aligned}
$$

The controllers' parameters are as follows:

$$
L^{(1)}=L^{(2)}=\left[\begin{array}{cccccc}
-10 & 2 & 3 & 2 & 3 & 0 \\
2 & -10 & 2 & 3 & 3 & 0 \\
3 & 2 & -10 & 3 & 2 & 0 \\
2 & 3 & 2 & -10 & 2 & 0 \\
3 & 2 & 3 & 2 & -10 & 0 \\
0 & 0 & 0 & 0 & 0 & 0
\end{array}\right] \text {, }
$$

other parameters are the same in system (52).

According to Theorem 4, system (55) can achieve synchronization by pinning control. Solving the LMIs in Theorem 4, we can obtain the fuzzy pinning control gain matrices as follows:

$$
\begin{aligned}
& D_{31}=\left[\begin{array}{cc}
6.7338 & 0 \\
0 & 9.7243
\end{array}\right], \\
& D_{32}=\left[\begin{array}{cc}
6.7872 & 0 \\
0 & 9.6380
\end{array}\right], \\
& D_{41}=\left[\begin{array}{cc}
4.2085 & 0 \\
0 & 5.8983
\end{array}\right], \\
& D_{42}=\left[\begin{array}{cc}
4.1814 & 0 \\
0 & 5.9448
\end{array}\right] .
\end{aligned}
$$

From the examples, network (55) without control is shown in Figure 1, and the system with pinning control is shown in Figure 2. It is easy to see that the results are very good by our methods. We also show the synchronization errors in Figure 3, where $e_{j}(t)=\left(z_{i j}(t)-z_{1 j}(t)\right)$, $i=2,3,4,5,6 ; j=1,2$.

\section{Conclusion}

This paper has investigated the synchronization of T-S FNNs by proposing a novel pinning control scheme. Instead of the presenting technique in prior, the proposed scheme regulates the column controllers by some events which are yielded by the relationships among the LKF and three nonnegative regions. Therefore, the traditional controllers have been improved as the eventdependent one in this paper. A concise criterion has been presented to guarantee the pinning synchronization of the considered CNNs. Simulations are finally provided to display the feasibility and improvements of the proposed pinning control scheme. Our results can only be studied as theoretical research now. We expect that the innovations of this paper can shed further light on the more problems (such as ([34-36]) under column controllers law. By the similar mechanism, our further directions include (1) design an intermittent output feedback controller and (2) design an intermittent adaptive controller.

\section{Data Availability}

The data used to support the findings the study are available from the corresponding author upon request. 


\section{Conflicts of Interest}

The authors declare that there are no conflicts of interest regarding the publication of this paper.

\section{Acknowledgments}

This work was supported by the National Defense Pre-Research Foundation of China (1126170104A, 1126180204B, and 1126190508A).

\section{References}

[1] R. Samli, "A new delay-independent condition for global robust stability of neural networks with time delays," Neural Networks, vol. 66, pp. 131-137, 2015.

[2] C. Yang, C. Chen, W. He, R. Cui, and Z. Li, "Robot learning system based on adaptive neural control and dynamic movement primitives," IEEE Transactions on Neural Networks and Learning Systems, vol. 30, no. 3, pp. 777-787, 2019.

[3] G. Peng, C. Yang, W. He, and C. L. P. Chen, "Force sensorless admittance control with neural learning for robots with actuator saturation," IEEE Transactions on Industrial Electronics, vol. 67, no. 4, pp. 3138-3148, 2020.

[4] C. Yang, G. Peng, L. Cheng, Na Jing, and Z. Li, "Force sensorless admittance control for teleoperation of uncertain robot manipulator using neural networks," IEEE Transactions on Systems, Man, and Cybernetics: Systems, pp. 1-11. in press, 2019.

[5] W. He, Y. Chen, and Z. Yin, "Adaptive neural network control of an uncertain robot with full-state constraints," IEEE Transactions on Cybernetics, vol. 46, no. 3, pp. 620-629, 2016.

[6] X. F. Wang and G. Chen, "Synchronization in small-world dynamical networks," International Journal of Bifurcation and Chaos, vol. 12, no. 1, pp. 187-192, 2002.

[7] H. Li, X. Liao, G. Chen, D. J. Hill, Z. Dong, and T. Huang, "Event-triggered asynchronous intermittent communication strategy for synchronization in complex dynamical networks," Neural Networks, vol. 66, pp. 1-10, 2015.

[8] J. Qin, H. Gao, and W. X. Zheng, "Exponential synchronization of complex networks of linear systems and nonlinear oscillators: a unified analysis," IEEE Transactions on Neural Networks and Learning Systems, vol. 26, no. 3, pp. 510-521, 2015.

[9] X. Chen, T. Huang, J. Cao, J. H. Park, J. Qiu, and J. Qiu, "Finite-time multi-switching sliding mode synchronisation for multiple uncertain complex chaotic systems with network transmission mode," IET Control Theory \& Applications, vol. 13, no. 9, pp. 1246-1257, 2019.

[10] F. Qu, J. Liu, H. Zhu, and B. Zhou, "Wind turbine fault detection based on expanded linguistic terms and rules using non-singleton fuzzy logic," Applied Energy, vol. 262, Article ID 114469, 2020.

[11] F. Qu, J. Liu, H. Zhu, and D. Zang, "Wind turbine condition monitoring based on assembled multidimensional membership functions using fuzzy inference system," IEEE Transactions on Industrial Informatics, vol. 16, no. 6, pp. 4028-4037, 2020.

[12] X. Yu, W. He, H. Li, and J. Sun, "Adaptive fuzzy full-state and output feedback control for uncertain robots with output constraint," IEEE Transactions on Systems, Man, and Cybernetics: Systems, pp. 1-14. in press, 2020.

[13] H. Lin, T. Zhang, Z. Chen, H. Song, and C. Yang, "Adaptive fuzzy Gaussian mixture models for shape approximation in robot grasping," International Journal of Fuzzy Systems, vol. 21, no. 4, pp. 1026-1037, 2019.

[14] J. Jian and W. Jiang, "Lagrange exponential stability for fuzzy Cohen-Grossberg neural networks with time-varying delays," Fuzzy Sets and Systems, vol. 277, no. 15, pp. 65-80, 2015.

[15] X. Yang and Z. Yang, "Synchronization of TS fuzzy complex dynamical networks with time-varying impulsive delays and stochastic effects," Fuzzy Sets and Systems, vol. 235, no. 16, pp. 25-43, 2014.

[16] Y. Liu, B.-Z. Guo, J. H. Park, S.-M. Lee, and S.-M. Lee, "Nonfragile exponential synchronization of delayed complex dynamical networks with memory sampled-data control," IEEE Transactions on Neural Networks and Learning Systems, vol. 29, no. 1, pp. 118-128, 2018.

[17] Z.-M. Zhang, Y. He, M. Wu, and Q.-G. Wang, "Exponential synchronization of neural networks with time-varying delays via dynamic intermittent output feedback control," IEEE Transactions on Systems, Man, and Cybernetics: Systems, vol. 49, no. 3, pp. 612-622, 2019.

[18] S. Ding and Z. Wang, "Lag quasi-synchronization for memristive neural networks with switching jumps mismatch," Neural Computing and Applications, vol. 28, no. 12, pp. 4011-4022, 2017.

[19] Y. Liang and X. Wang, "Synchronization in complex networks with non-delay and delay couplings via intermittent control with two switched periods," Physica A: Statistical Mechanics and its Applications, vol. 395, pp. 434-444, 2014.

[20] T. Chen, X. Liu, and W. Lu, "Pinning complex networks by a single controller," IEEE Transactions on Circuits and Systems I: Regular Papers, vol. 54, no. 6, pp. 1317-1326, 2007.

[21] X. Tianping Chen and T. Chen, "Cluster synchronization in directed networks via intermittent pinning control," IEEE Transactions on Neural Networks, vol. 22, no. 7, pp. 10091020, 2011.

[22] J. Zhou, J.-A. Lu, and J. Lü, "Pinning adaptive synchronization of a general complex dynamical network," Automatica, vol. 44, no. 4, pp. 996-1003, 2008.

[23] W. Yu, P. DeLellis, G. Chen, M. Di Bernardo, and J. Kurths, "Distributed adaptive control of synchronization in complex networks," IEEE Transactions on Automatic Control, vol. 57, no. 8, pp. 2153-2158, 2012.

[24] Y. Tang, H. Gao, J. Lu, and J. Kurths, "Pinning distributed synchronization of stochastic dynamical networks: a mixed optimization approach," IEEE Transactions on Neural Networks and Learning Systems, vol. 25, no. 10, pp. 1804-1815, 2014.

[25] Q. Chen and J. Cao, "Synchronization of complex dynamical networks with discrete time delays on time scales," Neurocomputing, vol. 151, pp. 729-736, 2015.

[26] J.-L. Wang, H.-N. Wu, and L. Guo, "Novel adaptive strategies for synchronization of linearly coupled neural networks with reaction-diffusion terms," IEEE Transactions on Neural Networks and Learning Systems, vol. 25, no. 2, pp. 429-440, 2014.

[27] J.-L. Wang and H.-N. Wu, "Synchronization and adaptive control of an array of linearly coupled reaction-diffusion neural networks with hybrid coupling," IEEE Transactions on Cybernetics, vol. 44, no. 8, pp. 1350-1361, 2014.

[28] Y. Liu, Z. Wang, and X. Liu, "Global exponential stability of generalized recurrent neural networks with discrete and distributed delays," Neural Networks, vol. 19, no. 5, pp. 667-675, 2006.

[29] Y. Liu, Z. Wang, X. Liang, and X. Liu, "Stability and synchronization of discrete-time markovian jumping neural networks with mixed mode-dependent time delays," IEEE 
Transactions on Neural Networks, vol. 20, no. 7, pp. 11021116, 2009.

[30] Y. Liu, Z. Wang, J. Liang, and X. Liu, "Synchronization of coupled neutral-type neural networks with jumping-modedependent discrete and unbounded distributed delays," IEEE Transactions on Cybernetics, vol. 43, no. 1, pp. 102-114, 2013.

[31] Y. Liu, Z. Wang, J. Liang, and X. Liu, "Synchronization and state estimation for discrete-time complex networks with distributed delays," IEEE Transactions on Systems, Man, and Cybernetics, Part B (Cybernetics), vol. 38, no. 5, pp. 1314-1325, 2008.

[32] D. Gong, H. Zhang, Z. Wang, and B. Huang, "Novel synchronization analysis for complex networks with hybrid coupling by handling multitude kronecker product terms," Neurocomputing, vol. 82, pp. 14-20, 2012.

[33] Z. Wang, Y. Wang, and Y. Liu, "Global synchronization for discrete-time stochastic complex networks with randomly occurred nonlinearities and mixed time delays," IEEE Transactions on Neural Networks, vol. 21, no. 1, pp. 11-25, 2010.

[34] C. Yang, Y. Jiang, W. He, J. Na, Z. Li, and B. Xu, "Adaptive parameter estimation and control design for robot manipulators with finite-time convergence," IEEE Transactions on Industrial Electronics, vol. 65, no. 10, pp. 8112-8123, 2018.

[35] C. Yang, H. Wu, Z. Li, W. He, N. Wang, and C.-Y. Su, "Mind control of a robotic arm with visual fusion technology," IEEE Transactions on Industrial Informatics, vol. 14, no. 9, pp. 3822-3830, 2018.

[36] W. He, C. Xue, X. Yu, Z. Li, and C. Yang, "Admittance-based controller design for physical human-robot interaction in the constrained task space," IEEE Transactions on Automation Science and Engineering, pp. 1-13. in press, 2020. 